\title{
La ablación genital femenina en comunidades emberá chamí
}

\author{
Raquel González Henao**
}

\section{Resumen}

Este artículo aborda el tema de la ablación genital femenina, que se realiza en algunas comunidades indígenas emberá chamí. Desde una perspectiva antropológica y retomando elementos del feminismo, se analiza la relación entre esta práctica, la construcción cultural de la identidad femenina, la regulación de la sexualidad y los mandatos sociales dirigidos a las mujeres.

Palavras-llave: Ablación, Cultura, Sexualidad, Emberá Chamí.

* Recebido para publicação em 07 de setembro de 2011, aceito em 19 de setembro de 2011.

** Antropóloga, candidata a magíster en filosofía de la Universidad Nacional de Colombia. Investigadora Independiente. r.gonzalezhenao@gmail.com

cadernos pagu (37), julho-dezembro de 2011:163-183. 
La ablación genital femenina

Female Genital Ablation in Embera Chami Communities

\begin{abstract}
This article approaches the topic of the genital feminine ablation. It is a practice realized by some indigenous communities embera chamí, who live in the western zone of Colombia. From an anthropologic perspective and using elements of feminism, this article analyzes the relation among ablation, the cultural construction of feminine identity, the regulation of sexuality and the social mandates directed to women.
\end{abstract}

Key Words: Ablation, Culture, Sexuality, Emberá Chamí. 


\section{Introducción}

El pueblo emberá pertenece al tronco cultural y lingüístico Chocó, conformado por indígenas waunana y emberá, originarios del territorio que actualmente corresponde al departamento del Chocó, al occidente de Colombia. ${ }^{1}$ Los emberá habitan mayoritariamente entre la cordillera central y el océano Pacífico; de acuerdo a las características de su territorio, se diferencian entre sí de la siguiente manera: emberá dobida, gente de río; emberá oibida, gente de selva; emberá pusabida, gente de mar, y emberá eyabida, gente de montaña. Estos últimos se dividen en dos grupos, catíos y chamíes. ${ }^{2}$

En el año 2007, Araceli Ocampo, funcionaria pública del municipio de Pueblo Rico, en el departamento de Risaralda, denunció la muerte de una niña emberá chamí recién nacida, cuyo deceso fue causado por la realización de la clitoridectomía. ${ }^{3}$

La noticia de la existencia de la ablación entre los emberá causó impacto en la opinión pública nacional, puesto que se consideraba que dicha práctica era exclusiva de algunas culturas

\footnotetext{
1 Colombia se divide política y administrativamente en 32 departamentos y un distrito capital. Cada departamento se encuentra conformado por varios municipios.
}

2 Además de los pueblos de tradición Chocó, en la actualidad también se identifican como emberá chamí, las comunidades indígenas del departamento de Caldas. Aunque en este territorio existen familias chamí en los municipios de San José, Risaralda, Belalcázar y Anserma, lo más probable es que la mayoría de la población indígena que habita el departamento, en los municipios de Riosucio, Supía, Marmato, Neira, Filadelfia y parte de Anserma, desciendan de los pueblos indígenas que al momento de la invasión española habitaban la vertiente del río Cauca, en lo que hoy corresponde a los departamentos de Caldas, Antioquia y Quindío.

3 Para mayor información sobre la denuncia y el desarrollo de este proceso se puede consultar la sistematización del proyecto Emberawera, realizada por Juanita Henao (2010).

[http://programacontraviolenciasdegenero.org/vbg_docum_publicac.php?id_clase _doc $=1]$. 
africanas y asiáticas. No se tenía certeza de su presencia en América, a pesar de que desde la década de los setenta se contaba con registros etnográficos que daban cuenta de su existencia entre los emberá chamí, y de que en la década de los noventa ya se había hablado públicamente de esta situación. ${ }^{4}$ De hecho, algunas organizaciones feministas y personal de salud conocía o intuía que la clitoridectomía era frecuente en las comunidades chamí de Risaralda, pero esto no había sido confirmado por las autoridades indígenas, ni se contaba con un caso documentado en esta materia (Henao, 2010).

La puesta en escena de este tema ha generado gran interés entre diferentes organizaciones, agencias de cooperación internacional, miembros de la academia y otros sectores de la sociedad civil, quienes han promovido investigaciones y procesos de incidencia política encaminados a dilucidar las dimensiones culturales, de derecho y de género de la práctica, y a proponer estrategias para su erradicación.

Con la intención de contribuir al debate, en este artículo se analiza el tema desde un punto de vista antropológico, se trata de comprender los significados y representaciones sociales entorno a la ablación, y estudiarlos en relación con los procesos de construcción de la identidad femenina.

Este documento está basado en una investigación sobre memoria histórica de las mujeres, que fue coordinada $y$ sistematizada por la autora, como parte del equipo de la fundación

\footnotetext{
4 El antropólogo Luis Guillermo Vasco (1975) señala que durante su trabajo de campo en la zona de El Chamí (Risaralda) un misionero mencionó la existencia de la clitoridectomía, sin embargo, esto no fue comprobado durante la etnografía que el investigador estaba realizando. Cayón y Gutiérrez (1981) documentan la existencia de la práctica en su etnografía de los emberá chamí, y Víctor Zuluaga y Patricia Granada (1997) exponen en un breve artículo los fundamentos míticos de la ablación genital femenina.
} 
Centro de Cooperación al Indígena-CECOIN, durante 2009 y $2011 .{ }^{5}$

Debido a que en Colombia la ablación genital femenina sólo ha sido objeto de investigación desde el año $2007^{6}$, el tema aún se encuentra en un estado inicial y la mayoría de la información no ha sido publicada. Por esta razón, las fuentes con las que se cuenta para este artículo son de carácter primordialmente testimonial.

A través de las voces de mujeres, nos introduciremos en los intersticios de esta práctica, para conocer no sólo de qué manera se realiza sino, también, el tejido cultural en el cual se encuentra inmersa, las representaciones sociales a las cuales remite y su relación con la configuración de la identidad de género entre las mujeres emberá chamí.

Los testimonios presentados fueron recogidos en conversaciones con mujeres indígenas que habitan en los departamentos de Risaralda, Antioquia y Caldas, ubicados al noroeste del país. Algunos de estos testimonios se refieren a

${ }^{5}$ CECOIN es una organización social colombiana fundada en la década de los ochenta, cuyo trabajo consiste en prestar asesoría y acompañamiento a los pueblos indígenas, y realizar procesos de investigación sobre la condiciones de vida de las comunidades, focalizando desde 2008 el conocimiento de la experiencia de las mujeres. Los resultados de la investigación sobre memoria histórica se recogen en el documento Por los Caminos de la memoria: Las mujeres embera chamí en la cultura, la política y la vida comunitaria. A la fecha de la redacción de este artículo, dicho material se encuentra en proceso de edición; podrá ser consultado a partir de noviembre de 2011. Es posible acceder a este documento escribiendo a cecoin@etb.net.co y r.gonzalezhenao@gmail.com.

${ }^{6} \mathrm{Al}$ momento se conocen cuatro investigaciones sobre el tema: i) La realizada por el fondo de Población de Naciones Unidas en asocio con el Consejo Regional Indígena de Risaralda-CRIR/ACIR, en el marco del proyecto Emberawera (20072010); la tesis de Sergio Estarita sobre derechos humanos y autodeterminación de los pueblos indígenas (2010); la investigación de CECOIN en la cual se basa este artículo (2011), y otra de Jorge Armando Triana, estudiante de psicología, mencionada por Juanita Henao en su sistematización del proyecto Emberawera (2010). 
hechos recientes, $y$ otros relatan experiencias ocurridas hace más de diez años en comunidades donde actualmente no se realiza la ablación. Curiosamente, son estas donde se recopiló mayor información al respecto, quizás porque al tratarse de un hecho del pasado existe mayor tranquilidad para mencionarlo.

Las palabras de las mujeres serán citadas sin indicar el lugar exacto en el cual fue obtenida la información, debido a que ellas autorizaron su uso con esta condición. ${ }^{7}$ Esta medida permite proteger su identidad, evita que sean estigmatizadas y que sus comunidades se prevengan frente a futuros diálogos $e$ investigaciones sobre el tema.

\section{La ablación como práctica cultural}

Cuando se pregunta a las emberá chamí sobre cómo deben comportarse para ser consideradas buenas mujeres dentro de sus comunidades, ellas señalan algunas características que pueden resumirse en los siguientes mandatos: ser fieles a su pareja, cuidar de los hijos, participar en las actividades de la comunidad sin tener problemas con las demás personas, hablar el idioma propio y, en general, mantener y defender las costumbres de los emberá. Estas características enunciadas por ellas dan cuenta del rol que socialmente se les asigna a las mujeres y que consiste, básicamente, en la reproducción de la cultura y el cuidado de la familia.

La construcción del comportamiento socialmente aceptado para hombres y mujeres se da a partir de diferentes mecanismos,

\footnotetext{
7 Las mujeres fueron consultadas sobre el uso de sus testimonios con el fin de promover la reflexión sobre la vida de las mujeres $y$, específicamente, sobre la curación. Dicha consulta se realizó en el mismo momento en que se realizaron las entrevistas y durante el Encuentro de Mujeres Emberá Chamí de Antioquia, Caldas y Risaralda, que se llevó a cabo en el municipio de Riosucio, Caldas, durante el 2010. Las emberá chamí señalaron que era importante hablar sobre lo que les pasaba como mujeres y solicitaron que en este tema se guardara la identidad de quienes habían hablado al respecto.
} 
que incluyen enseñanzas por parte de las personas mayores, uso de la medicina tradicional y realización de ritos de paso. ${ }^{8}$ Con éstos se marcan las distintas etapas del ciclo vital y se refuerzan los conocimientos que es necesario adquirir en cada uno de estos momentos. Tales saberes son fundamentales para introducirse totalmente en la cultura, garantizar la propia subsistencia y ser capaces de cumplir con las tareas asignadas dentro de la comunidad.

El aprendizaje de los roles de género para las mujeres emberá chamí comienza desde los primeros días de vida y culmina durante la pubertad, momento en el cual se espera que la niña que se convierte en mujer tenga las habilidades suficientes para "hacer destino", es decir, para asumir sus responsabilidades como emberá, especialmente, aquellas ligadas a la familia.

Las destrezas y saberes incorporados durante años son reforzados a lo largo de la vida, durante la práctica cotidiana y por medio de dispositivos a través de los cuales la sociedad establece si se está cumpliendo con lo esperado y controla los desvíos del comportamiento aceptado. Estos dispositivos incluyen desde el rechazo de la comunidad hasta actos de violencia física.

Dentro de su proceso de construcción como mujeres, uno de los ritos establecidos por la cultura emberá chamí es la ablación del clítoris. Esta práctica se ha identificado en comunidades chamí de Risaralda y se tienen registros de que algunas otras de Caldas y Antioquia la mantuvieron en algún momento. No existe un reconocimiento público de las autoridades indígenas sobre la realización de la clitoridectomía en otros departamentos, aunque es probable que ocurra, dada la cercanía cultural y geográfica entre los diferentes grupos emberá.

8 Se toma la categoría rito de paso propuesta por el antropólogo Arnold van Gennep para referirse a las diferentes ceremonias que en determinada cultura se utilizan para marcar el tránsito que las personas hacen de una etapa a otra de su vida. 
Las chamí dan diferentes nombres a la ablación; la manera más frecuente de llamarla es "curación", pero también se utilizan otras expresiones para referirse a ella, como "el arreglo" o "la operación". Dichas denominaciones dan cuenta del significado que se otorga al procedimiento, que se supone consiste en arreglar, operar o curar un defecto en el cuerpo de las mujeres.

Eso decía mi mamá, que una niña nacía y que en la vagina tenía una cosa con pelitos encima, con unas telitas por lado y lado, y que eso lo operaban [...] Y ya, ponían un poquito de alcohol y ya con eso ya iba sanando. Eso me decía mi mamá, ella me enseñó. Pero ya no lo hago (Testimonio de mujer emberá chamí, 2009).

De acuerdo a lo planteado por las indígenas chamí en sus testimonios, la ablación consiste en hacer un pequeño corte en el clítoris cuando se considera que éste tiene un tamaño mayor de lo normal. Dicho procedimiento, se realiza cuando la niña tiene entre dos y quince días de nacida. En uno de los testimonios recopilados se habla de un corte que ocurrió durante la juventud, pero éste constituye un caso excepcional y no fue posible documentarlo debido a que la persona que aportó el testimonio no logró continuar con la narración porque le producía gran aflicción pensar en ello.

En esta cultura, "la curación" se explica proporcionando varias razones, algunas de ellas son comunes a todos los relatos, otras solo aparecen en las narraciones de algunas mujeres. A pesar de estas diferencias, el punto de partida es el mismo: la idea según la cual el clítoris es normal cuando tiene un tamaño pequeño, pero cuando sobresale constituye un defecto de nacimiento, que si no se corrige a tiempo conllevará graves consecuencias para la mujer, su familia y su entorno social.

En ciertas comunidades, según la creencia que se ha trasmitido por varias generaciones, si no se realiza la ablación, el 
clítoris sigue creciendo hasta parecerse a un pene o hasta alcanzar un tamaño que en el momento del acto sexual resulta incómodo. Esto causa dificultades que pueden nombrarse de la siguiente manera:

i) Estéticas: puesto que las mujeres consideran que si el clítoris es demasiado grande "se ve feo".

ii)Sociales: porque la mujer comenzaría a desear el placer sexual con tanta frecuencia como lo hacen los hombres. Algunas mujeres señalaron que la niña que no es "operada" puede llegar a sentir atracción por otras mujeres.

iii) Familiares: porque se supone que los hombres no toman por esposa a una mujer cuyo clítoris sea "demasiado grande". Así lo explica una de las emberá:

Cuando la niña nace con el clítoris largo, que sobrepasa la vulva, se arregla para que no crezca como un pene. La curación es para evitar que en el momento de tener relación sexual con el marido no se ponga erecto como el pene, porque eso muchas veces no le gusta a los hombres (Testimonio de mujer emberá chamí, 2010).

Cuando comenzaron las indagaciones sobre la ablación genital entre los chamí, se difundió la versión según la cual el corte del clítoris se realizaba para evitar que las mujeres sintieran placer durante el coito $y$ no se movieran. De acuerdo con lo documentado por Zuluaga y Granada (1997), se pensaba que si permanecían quietas durante la relación sexual, estaban colaborando para que Karagabí, el dios máximo de los emberá, pudiera sostener el mundo, sin que se produjeran desequilibrios.

Aunque esta explicación retoma elementos importantes de la mitología ancestral chamí, dicha referencia no apareció en las narraciones de las mujeres que fueron entrevistadas. Esto puede 
deberse a que se haya perdido la memoria de este relato, o que se trate de una justificación poco difundida.

La ablación es una práctica que sólo pueden llevar a cabo las mujeres, porque se encuentra dentro de su ámbito de conocimiento, específicamente de aquellas que ejercen como parteras. Muestra de ello es que en el momento en que se abrió el debate público sobre el tema algunos hombres líderes chamí señalaron que no sabían que esto pasaba en sus comunidades. Si bien desde hace cuatro décadas que se conoce de la existencia de la ablación, es muy probable, efectivamente, que los varones no tuvieran certeza de la misma, dado que se trata de una práctica que se realiza sin pedir su consentimiento.

A pesar de que es un rito que se ha instalado en la cultura emberá y que se considera fundamental para el desarrollo de las mujeres, el procedimiento es totalmente secreto. A diferencia de lo que ocurre con otros ritos de paso en los que participa toda la comunidad, de la "curación" solo se enteran la madre, la abuela y la partera que la realiza. En algunos casos, ni siquiera la madre participa del mismo, puesto que ocurre cuando ésta aún se encuentra en recuperación luego del parto.

La mayoría de mujeres con las cuales se conversó sobre el tema manifestaron no conocer esta práctica sino hasta el momento en que tuvieron su primera hija, incluso, no sabían si a ellas mismas se les había practicado la ablación. Otras señalaron que no habían escuchado nada al respecto porque sólo tenían hijos varones.

No, nosotros no sabíamos de eso, nosotros éramos inocentes de que eso existía aquí en la comunidad, pues como yo no tengo niñas, yo tampoco sabía cómo era el clítoris. [...] Yo me preguntaba por qué yo no tengo eso, entonces le preguntaba a mi mamá y ella me decía que yo había nacido así... Es que los mayores eran muy ocultos con esas cosas, ellos nunca le decían a uno nada, ni los hombres sabían (Testimonio de mujer emberá chamí, 2009). 
Para las emberaweraras ${ }^{9}$ el momento en el cual sus hijas pasan por el procedimiento de la ablación es desconcertante y muy fuerte emocionalmente. En sus narraciones se deja ver que ellas no comprendían bien lo que estaba ocurriendo ni por qué se realizaba. A pesar de ello, siguieron las indicaciones de las mayores, como muestra el siguiente testimonio:

Mire, yo misma quería ver cómo mi mamá y mi abuela hacían la curación a la niña mía, porque yo no sabía qué era eso. Pero mi mamá me dijo que no, que yo no podía ver, y yo le dije: '¿pero por qué yo no puedo ver?, ¿qué es lo que ustedes van a hacer a mi hija?', y ella me preguntó que si a mí no me daba pesar. Yo dije que no, yo quería ver cómo hacían curación a la niña mía. Yo no sabía [...] Entonces empezaron, yo pensaba: "¿yo qué hice?". Yo no era capaz de seguir. Ya a lo último me retiraron porque yo estaba muy mal y no dejaba (Testimonio de mujer emberá chamí, 2009).

Los detalles sobre cómo se realiza la ablación y las explicaciones de por qué se considera necesaria se trasmiten generacionalmente. Cuando se preguntó sobre las razones para hacer el procedimiento, las mujeres respondieron con frases como: "así decían los antiguos", "eso lo aprendí de mi mamá", "esa es costumbre de nosotros los emberá". Casi siempre es la abuela quien comunica a la madre que debe practicarse el "arreglo", ya sea que lo asuma directamente la anciana cuando tiene conocimientos de partería o se busque a una partera de la comunidad.

Hay unas mujeres que las operaban. Mi mamá arreglaba a mi niña, me decía que había que arreglarla, me decía: 'hija póngale cuidado y verá que eso lo arregla es así'. La primera vez yo no sabía, claro que me dio miedo. Primero

${ }^{9}$ La palabra emberawera significa mujer emberá, y emberaweraras es su plural. 
tuve un embarazo de un niño, después fue una niña. Mi mamá dijo: "tienen que operar la niña". Decía que así no se veía nada más, que ya quedaba normal la vaginita (Testimonio de mujer emberá chamí, 2009).

En las conversaciones con las mujeres sobre el tema, es evidente que no están de acuerdo con la práctica porque les parece dolorosa y violenta. La mayoría de las emberá entrevistadas dijeron que ellas no deseaban aprender y que experimentaron angustia porque no lograron evitar el procedimiento. Sin embargo, se encontraban en una disyuntiva, dado que al mismo tiempo que reprobaban la ablación tenían la presión social de quienes señalaban que si no se realizaba, su hija llevaría una vida anormal, sería rechazada por los hombres y podría llegar a sentir deseo sexual hacia otras mujeres.

Después de saber de la existencia de la "curación", buena parte de ellas la practicó a sus demás hijas, otras, por el contario, no permitieron que se hiciera la ablación a sus niñas, aunque tenían, y sigan teniendo, dudas sobre las consecuencias de esta decisión. Esto se hace evidente en el siguiente testimonio, proporcionado por una mujer emberá chamí que vive en una de las comunidades donde ya no se práctica la ablación desde hace aproximadamente una década:

Hay una compañera mía, ella me contaba que a su hija mayor también le hicieron y a la menor no, y ella dice: '¿será que es cierto lo que decían los abuelos? A esa muchachita no la arreglé, la dejé así. Y esa muchacha se alborota por todo [tiene una vida sexual activa]. En cambio la grande, a la que arreglaron, anda un poquito calmadita'. Entonces yo le decía: 'iAy! si usted dice eso, ahora la mía también le va a pasar lo mismo. Hasta ahora es normal, ella está en la casa, ella está viendo televisión. Hasta ahora yo veo que está juiciosa, ¿qué tal más adelante?'. Entonces yo digo: ¿será que vamos a tener que ver un problema por 
algo que dejamos sin arreglar? (Testimonio de mujer emberá chamí, 2009).

En algunas comunidades emberá chamí, curiosamente aquellas en las cuales se han dejado de practicar otras ritualidades, la ablación fue suspendida debido a experiencias traumáticas para la comunidad y para las mujeres, como la muerte de niñas o el hecho de que los hombres se hubiesen dado cuenta de que este procedimiento se realizaba.

Eso es como un tabú que no se le cuenta a los hombres, hasta que una vez a una señora se le murió una niña de tres días de nacida. Una señora mayor llegó y le practicó ese trabajo a las nueve de la mañana y cuando el papá llegó en la tarde la niña había fallecido a las dos de la tarde. Empezó a haber chisme entre las mayoras y por eso se supo, para ellas era un secreto pero los compañeros se enteraron por los chismes y pensaron que eso era malo, que eso era delito (Testimonio de mujer emberá chamí, 2010).

Aunque ya no se hace el corte, en ciertos lugares se han implementado procedimientos que, se supone, garantizan que las niñas se convertirán en buenas mujeres. Por ejemplo, en una comunidad se utiliza la práctica de frotar el clítoris de la recién nacida con un huevo de gallina tibio para disminuir su tamaño, como cuenta esta emberawera:

Entonces ya fue uno pensando, allí mucha gente decía: "hombre, con unos huevos, apenas se ponen calienticos, ahí mismo sobarle allá". Cuando la gallina coloca el huevo, ahí mismo hay que cogerle y con eso sobarle allá en la vagina. Si eso no se hace, cuando ya va creciendo la niña, cuando tenga un añito, entonces se va alborotando más, por eso no se puede dejar eso así. [...] Ahora eso [ablación] no se hace pero lo del huevo sí (Testimonio de mujer emberá chamí, 2009). 
En otras comunidades, donde hasta el 2010 se realizaba la ablación pero se estaba reflexionando sobre la importancia de abandonar esta práctica, las mujeres planteaban la necesidad de investigar si era posible reemplazar el corte del clítoris por otros procedimientos en los cuales se utilizarán únicamente plantas medicinales, para no causar dolor ni poner en riesgo la vida ni la salud de las niñas.

El interés en encontrar estas alternativas que buscan reemplazar la ablación, muestra que, ciertamente, se reprueba el hecho concreto de cortar el clítoris, sin embargo, los imaginarios culturales que sustentan la "curación" permanecen.

Persisten representaciones sociales desde las cuales se limita la libertad y la autonomía de las mujeres sobre su cuerpo, dado que la cultura continúa delegando en otras y otros el control de la sexualidad.

Así pues, en el proceso general de construcción de la identidad de género, la ablación se erige como mecanismo para asegurar la forma correcta de vivir el placer y el deseo, con miras a garantizar lo que se considera el funcionamiento adecuado de la pareja y de las relaciones erótico-afectivas entre hombres $y$ mujeres, basándose en concepciones hetero-normativas y patriarcales.

Este ejercicio de control sobre el cuerpo de las mujeres, no es agenciado únicamente por los varones, pero los sitúa en un lugar de poder superior, puesto que promueve una dinámica del deseo sexual que esté siempre dispuesta al requerimiento masculino, que no se exprese de manera autónoma por parte de las mujeres y que se dirija exclusivamente hacia sus compañeros sentimentales, no hacia otros hombres $y$, mucho menos, hacia otras mujeres.

A partir de lo anterior, es posible afirmar que si realmente se quiere transformar la ablación, evitar que se retome en comunidades donde ya no se realiza y prevenir la aparición de nuevas prácticas de control del cuerpo de las mujeres, es preciso cuestionar los ideales de "buen comportamiento" que le dan 
sentido a la ablación como rito de paso necesario. Se requiere la construcción de nuevos referentes culturales, donde el cuerpo y la sexualidad femenina sean comprendidos como esferas de dominio autónomo de las mujeres.

\section{El abandono de la ablación genital femenina}

A partir de la denuncia pública sobre la existencia de la ablación genital femenina entre los emberá chamí, hubo diferentes manifestaciones de rechazo por parte de la sociedad, por parte de las defensoras de derechos humanos y las organizaciones de mujeres y feministas.

Se colocó el tema en el centro del debate y, en ocasiones, hasta se transmitió la idea de que los indígenas eran salvajes y atrasados, por ejemplo, en el 2008 se emitió el programa de televisión Séptimo Día, en el cual se presentó el tema de la ablación de una manera que para las comunidades fue agresiva y descontextualizada.

Además, se promulgó una sentencia por parte del Juzgado Promiscuo Municipal de Pueblo Rico, en la cual se señaló que la ablación es "una práctica bárbara, inhumana, violatoria de los derechos de la mujer y de las niñas de esa comunidad [emberá chamí]", y se cuestionaron las acciones pedagógicas que hasta ese momento habían adelantado las autoridades indígenas, el Ministerio del Interior y de Justicia, la Defensoría del Pueblo y el Instituto Colombiano de Bienestar familiar - ICBF, frente a la ablación, diciendo que eran "insuficientes, ineficaces, lentas, tolerantes e inocuas para defender la vida e integridad personal de las niñas de la comunidad indígena emberá-chamí" ${ }^{10}$

La sentencia motivó la atención de diferentes sectores, convocó a actuar al respecto e hizo visible de que no se trataba de un caso de violencia intrafamiliar sino de un hecho cultural. Sin embargo los calificativos utilizados para referirse a una práctica

${ }^{10}$ Sentencia proferida el 24 de julio del 2008. 
que hace parte del ciclo vital de las mujeres emberá, generaron una gran prevención al interior de las comunidades. Aunque las mujeres se cuestionaban sobre la práctica, y parte de ellas no la aprobaba, sintieron vulnerada su intimidad y atacada su identidad como indígenas:

(...) Por ese escándalo que salió a la luz pública nosotros dentro de la comunidad de Mistrató... muchas compañeras cuando íbamos a tener parto, nosotros éramos bien reservadas, que los niños, jóvenes, menores de edad no vieran como nacer un bebé. Pero después de haber salido eso a la luz pública los niños en la calle ya saben cómo nace un niño, cómo maneja posición de la mamá y eso quiere decir para nosotros que los niños están escandalizados (...) A nosotros nos tiraron agua sucia a nuestra comunidad, en vez de haber ido a orientar con los dirigentes y con las dirigentes de la comunidad, debieron sensibilizar a la comunidad. Así han escandalizado a nuestros hijos (Testimonio de mujer emberá chamí, 2010).

Entre las iniciativas de carácter pedagógico impulsadas por las diferentes entidades públicas y privadas que han intervenido para promover la erradicación de la ablación, una de las experiencias más conocidas fue el proyecto Emberawera, desarrollado desde el 2007 por el Fondo de Población de las Naciones Unidas - UNFPA, en asociación con el pueblo emberá chamí de los resguardos indígenas de Mistrató y Pueblo Rico, el Consejo Regional Indígena de Risaralda $(\mathrm{CRIR})^{11}$ e instituciones del gobierno nacional y departamental. ${ }^{12}$

Gracias a la reflexión iniciada en el marco del proceso, en el 2009 el Consejo Regional Indígena de Risaralda emitió una resolución señalando que se suspendía la práctica durante dos

\footnotetext{
${ }^{11}$ Recientemente el CRIR cambió su figura legal y se constituyó como Asociación de Cabildos Indígenas de Risaralda-ACIR.

${ }^{12}$ Para mayor información, ver: http://www.unfpa.org.co/menuSuplzqui.php?id=26
} 
años, mientras se tomaba el tiempo para hacer una reflexión profunda al respecto. ${ }^{13}$ Posteriormente, el 23 de noviembre de 2010, las autoridades indígenas de Risaralda anunciaron la suspensión definitiva de la práctica.

En consonancia con esto, mujeres, comunidades y líderes han dicho públicamente que la ablación no se realiza y hablan de ella como un asunto del pasado. A pesar de esto, a comienzos del 2011, Contigo Mujer, organización feminista de Risaralda, hizo un llamado de alerta debido a un nuevo caso de ablación practicado en una niña de siete días de nacida ${ }^{14}$, lo que demuestra que el abandono de la ablación es un cambio que puede tardar mucho más.

El hecho de que aún se mantenga la práctica, a pesar del debate colectivo que ha realizado el pueblo emberá chamí y del pronunciamiento de sus autoridades, convoca a una reflexión sobre las estrategias de intervención y sobre el tiempo que toman los procesos de cambio cultural, tristemente lentos, si se recuerda que mientras se pasa de reflexión a la acción, muchas niñas emberá serán "operadas".

El análisis de este tema es inseparable de la tensión entre derechos individuales y derechos colectivos, expresada claramente por el investigador Sergio Estarita, quien plantea:

\begin{abstract}
(...) Se podría afirmar que la discusión se centra en la tensión de dos derechos, los de la comunidad o resguardo indígena como colectividad, especialmente la autonomía y autodeterminación de los pueblos y comunidades indígenas, y por otro lado, los derechos humanos o fundamentales de las niñas y mujeres indígenas, como derechos individuales o subjetivos (Estarita, 2010:4).
\end{abstract}

\footnotetext{
${ }^{13}$ Resolución 001 del 127 de junio de 2009 para los municipios de Pueblo Rico y Mistrató. Consejo Regional Indígena de Risaralda.

${ }^{14} \mathrm{En}$ : http://corporacioncontigomujer.blogspot.com/2011/02/alerta.html
} 
Este conflicto entre derechos que genera el tema de la ablación hace difícil tomar una posición radical al respecto. Por supuesto, no se trata de defender la práctica, pero sería demasiado ingenuo creer que prohibiéndola o sancionando a las mujeres que la realizan se va a lograr su erradicación.

Quizás resulte más fácil abordar este tema trasladándolo al terreno de la cosmovisión chamí. Como en la mayoría de los pueblos indígenas de Colombia, desde la concepción emberá, el cosmos se organiza por parejas, tales como día y noche, hombre y mujer, frío y cálido. Los componentes de cada una de estas díadas, aparentemente opuestos, en realidad se complementan en una relación de equilibrio, ninguno está por encima del otro, ambos se necesitan para lograr la armonía y el funcionamiento adecuado de las comunidades.

Desde el ideal expresado en la cosmovisión, si bien se trata de seres distintos, hombres y mujeres desempeñan trabajos necesarios para la vida comunitaria y deben ser reconocidos, respetados y apoyados.

Retomando estos principios, vemos que no existe una diferenciación tajante entre el ser individual y la colectividad a la que se pertenece, por el contrario, del bienestar y del equilibrio entre cada uno de los seres que hace parte del territorio depende la armonía comunitaria, la cual, a su vez, es necesaria para que cada uno esté bien.

Cuando se revisa el caso de la ablación genital femenina desde esa perspectiva, finalmente, se llega a la conclusión de que dicha práctica violenta a las mujeres y desarmoniza su lugar en el cosmos. Así, la necesidad de transformación no obedece exclusivamente a una presión externa de la sociedad mayoritaria, sino que se arraiga fuertemente en la misma experiencia de las indígenas, para quienes la ablación es una práctica dolorosa y perjudicial que se realiza para responder a los imaginarios $e$ imposiciones sociales sobre el deber ser femenino.

En ese orden de ideas, el abandono de la práctica no sólo es posible sino necesario. Se trata, sin duda, de un reto importante, 
una transformación cultural a partir de la elaboración de nuevos referentes sobre la sexualidad femenina y de los elementos que configuran la identidad de género de las mujeres. Éste es un paso importante en el fortalecimiento de la autonomía de ellas, lo cual es condición necesaria para cambiar otras situaciones de violencia que viven en sus comunidades y frente a las cuales han levantado su voz de protesta.

Ahora bien, para comprender los posibles caminos que puede tomar dicha dicha transformación es preciso no perder de vista el contexto social y cultural específico emberá chamí. No podemos olvidar que las emberá, además de luchar por sus derechos como mujeres, son parte de un pueblo indígena que defienden y valoran y al cual están ligadas "como la mata de plátano está sembrada en la tierra" ${ }^{15}$, tal como lo expone esta emberawera:

Yo estoy en dudas y me he sentido apenada de esas tradiciones. Pero también pienso que nuestras madres han dado vida y de ahí nosotros vivimos en nuestros territorios tradicionales. Nosotros hemos venido milenariamente pisamos la madre tierra, y en este momento yo estoy aquí parada en nombre de mi tradición y de las parteras que me dieron la vida (Testimonio de mujer emberá chamí, 2010).

Las emberá enfrentan muchas otras situaciones que menoscaban sus posibilidades de pervivencia, como mujeres $y$ como indígenas. Son víctimas de diversas formas de violencia tanto por la acción de actores externos, como al interior de sus comunidades debido a la reproducción de prácticas de subordinación que se han incorporado a la cultura. La ausencia de condiciones de vida digna para ellas hace muy difícil su fortalecimiento $\mathrm{y}$, como consecuencia de ello, limita los procesos de transformación de imaginarios patriarcales.

${ }^{15}$ Expresión utilizada por una emberá chamí, 2011. 
Nuestras hijas son víctimas de violencia seria en nuestro territorio (...) La mujer siempre ha sido discriminada, también entre nosotras mismas. A las mujeres no les gusta que otras vayan a fortalecerse dentro de sus comunidades, no les gusta que las niñas sean educadas. Sólo nos gusta que cuiden a los hermanitos, no más. Entre nosotros mismos los indígenas hemos utilizado el abuso verbal, el abuso físico, el abuso de confianza, por eso no nos hemos fortalecido. Hay atropello por parte de actores armados que vienen a nuestros territorios (Testimonio de mujer emberá chamí, 2010).

A partir de lo anterior, es fundamental reconocer que para que sea posible y sostenible la transformación de la violencia que viven y de las prácticas que las vulneran, es preciso construir propuestas integrales de pervivencia de los pueblos.

\section{Referências bibliograficas}

CAYÓN, Edgardo y GuTIÉRREZ, Idelfonso. Etnografía de los Embera Chamí de Risaralda (Colombia). Popayán, Universidad del Cauca. Facultad de Humanidades. Departamento de Investigaciones Sociales, 1981.

ESTARITA, Sergio. Ablación en Colombia, ¿Derechos humanos o autodeterminación de los pueblos indígenas? Necesidad de una política pública y social de inclusión y reconocimiento de los derechos de las mujeres indígenas. Tesis de maestría, Política Social, Pontificia Universidad Javeriana, 2010.

GONZÁlez HENAO, Raquel. Por los caminos de la memoria: Las mujeres emberá chamí en la cultura, la política y la vida comunitaria. Bogotá, Centro de Cooperación al Indígena-CECOIN, 2011. [En proceso de publicación].

HENAO, Juanita. El proyecto Embera wera: Una experiencia de cambio cultural para la eliminación de la ablación genital femenina y la promoción de los derechos de mujeres Embera de los municipios de Mistrató y Pueblo Rico del departamento de Risaralda. Bogotá, 2010. 
En:

http://programacontraviolenciasdegenero.org/vbg_docum_publicac.ph p?id clase doc $=1$.

VASCO URIBE, Luis Guillermo. Los Chamí. La situación del indígena en Colombia. Bogotá, Editorial Margen Izquierdo, 1975.

Zuluaga GómezUluAGA GÓMEZ, Víctor y GRANAdA ECHEVERRY, Patricia La ablación del clítoris y su fundamento mítico. Revista de Ciencias Humanas. Universidad Tecnológica de Pereira, vol. 4, n 11. Pereira, 1997, pp. 47-51. 